\title{
A NOTE ON LIE GROUPS
}

\author{
HANS SAMELSON
}

1. Introduction. The following theorem, which plays a role in the classification of Lie groups, was first proved by $\mathrm{H}$. Weyl $[1,2]:^{1}$

THEOREM A. If $G$ is a real compact connected semi-simple Lie group, then any connected group $G^{\prime}$ locally isomorphic with $G$ is also compact.

It is well known and easily seen by considering the simply connected covering group that Theorem A can also be formulated as follows:

THEOREM B. The fundamental group of a real connected compact semi-simple Lie group is finite.

In this note we present two proofs of Theorems A and B; one proof uses differential forms, the other, which is somewhat more elementary, is based on differential geometry. ${ }^{2}$

Let then $G$ be a real connected compact Lie group and assume that the fundamental group of $G$ is infinite. We have to prove that $G$ is not semi-simple. We note that for compact groups "semi-simple" means that the center of $G$ is finite [2, p. 282].

2. Proof by differential forms. Since for group manifolds the fundamental group and the one-dimensional homology group coincide, our assumption means that the one-dimensional Betti number is not 0 . Let $Z$ denote a 1 -cycle, which is not homologous to 0 (with rational or real coefficients). By de Rhams theorem there exists an exact differential form $\omega$ of degree one such that $\int_{Z} \omega \neq 0$. It is well known from Cartan's investigations that we can replace $\omega$ by a form $\bar{\omega}$ which is invariant under the right and left translations of $G$. We denote by $a \cdot \theta$ resp. $\theta \cdot b$ the transform of the differential form $\theta$ under left resp. right translation so that $a \cdot \theta(x, d x)=\theta(a \cdot x, a \cdot d x)$, where $a \cdot x$ means the group product of the elements $a$ and $x$ of $G$ and $a \cdot d x$ means the image of the vector $d x$ under the left translation by $a$, and similarly for $\theta \cdot b$. With Haar measure on $G$ we form the expression $\bar{\omega}=\iint_{G} a \cdot \omega$ $\cdot b d a d b$; this is an invariant form on $G$ of degree 1 . We consider now

Received by the editors May 6, 1946.

${ }_{1}^{1}$ Numbers in brackets refer to the references cited at the end of the paper.

2 The first proof has also been known to C. Chevalley and G. de Rham for some time, and is given here mainly for completeness' sake. 


$$
\int_{Z} \bar{\omega}=\iint_{G} \int_{Z} a \cdot \omega \cdot b d a d b .
$$

By the formula for transformation of integrals we have $\int_{z} a \cdot \omega \cdot b$ $=\int_{a} \cdot z \omega \cdot b$, where $a \cdot Z$ is the image of $Z$ under left translation by $a$. But $a \cdot Z$ is homotopic to $Z$ since $a$ can be connected with the unit element $e$ by a continuous curve. Therefore $\int_{z} a \cdot \omega \cdot b=\int_{a} \cdot z \omega \cdot b=\int_{z} \omega \cdot b$, and by the same reasoning on $b$ we find $\int_{z} a \cdot \omega \cdot b=\int_{z} \omega$, and therefore finally

$$
\int_{Z} \bar{\omega}=\iint_{G} \int_{Z} \omega d a d b=\int_{Z} \omega \cdot \iint_{G} d a d b=\int_{Z} \omega \neq 0 .
$$

The form $\bar{\omega}$ is in particular invariant under the inner automorphisms $a^{-1} \cdot x \cdot a$ of $G$. Considering $\bar{\omega}$ at the unit element $e$ we have then a nonzero linear function on the tangent space at $e$ which is invariant under the linear transformations of the adjoint group. Since $G$ is compact we can introduce in the tangent space at $e$ an inner product which is invariant under the adjoint group. In a space with an inner product a linear function can be identified with a vector and so $\bar{\omega}$ gives us a vector at $e$ invariant under the adjoint group. (If we write $\bar{\omega}(e, d x)=\sum \alpha_{i} d x_{i}$ and assume that the adjoint group is represented by orthogonal matrices, this is simply the vector with components $\alpha_{i}$.) But then the one-parameter subgroup in direction of this vector is invariant under the adjoint group also, and lies therefore in the center of $G$, which shows that $G$ is not semi-simple.

3. Proof by differential geometry. The second proof rests on the consideration of geodesics. We assume again that the fundamental group of $G$ is infinite. We introduce in $G$ an invariant differential geometry; this is possible since $G$ is compact; "invariant" means that the right and left translations are isometries. It is well known that the geodesics going through $e$ are the one-parameter subgroups.

Let $\bar{G}$ be the simply connected covering group of $G$; we introduce the "covering" differential geometry on $\bar{G}$ by requiring that the local isomorphism between $G$ and $\bar{G}$ be an isometry. This differential geometry will also be invariant. Because of the assumption on the fundamental group, $\bar{G}$ is not compact.

As covering space of a compact space, $\bar{G}$ is a "complete" Riemannian space; any two points in it can be connected by a shortest geodesic, that is, by one which realizes the absolute minimum of curve length between the two points (see $[3,4,5]$ ). In $\bar{G}$ there exist therefore arbitrarily long geodesic segments which are the shortest con- 
nections of their end points. By moving the midpoint of each such segment to $\bar{e}$ (the unit element of $\bar{G}$ ) by means of a left translation, and by considering the limit of a properly chosen sequence we can find a geodesic $\bar{\gamma}$ through $\bar{e}$, which is a "straight line," that is, which realizes the shortest distance in $\bar{G}$ between any two of its points. We shall prove that $\bar{\gamma}$ belongs to the center of $\bar{G}$.

Consider the image $\gamma=c(\bar{\gamma})$ in $G$ of $\bar{\gamma}$ under the covering mapping $c: \bar{G} \rightarrow G$. The group $\gamma$ may or may not be closed; the closure of $\gamma$ is a connected compact Abelian Lie group, therefore a torus group $T$ of a certain dimension. We introduce arclength $s(-\infty<s<+\infty)$ on $\bar{\gamma}$, and write $\bar{\gamma}(s)$ for the point on $\bar{\gamma}$ with parameter value $s$; we can assume $\bar{\gamma}(0)=\bar{e}$.

We determine now a sequence $s_{n}$ of values of $s$, such that $s_{n} \rightarrow+\infty$, and $c\left(\bar{\gamma}\left(s_{n}\right)\right) \rightarrow e$. This is possible since $T$ is compact. From a certain $n$ on we can find points $\bar{e}_{n}$ in $\bar{G}$ such that (1) $c\left(\bar{e}_{n}\right)=e$ and (2) $d\left(\bar{\gamma}\left(s_{n}\right), \bar{e}_{n}\right)=d\left(c\left(\bar{\gamma}\left(s_{n}\right)\right), e\right.$ ) (where we denote by $d$ the distance in $G$ and in $\bar{G})$; this is possible because the covering mapping $c$ is a local isometry. It could happen that $\bar{\gamma}\left(s_{n}\right)=\bar{e}_{n}$. The points $\bar{e}_{n}$ are in the center of $\bar{G}$, as (1) shows.

Now let $a$ be any element of $\bar{G}$, and consider the transform $\bar{\delta}=a^{-1} \cdot \bar{\gamma} \cdot a$ of $\bar{\gamma}$; transformation by $a$ being an isometry the parameter $s$ on $\bar{\gamma}$ can also be used as arclength on $\bar{\delta}$. Suppose now that $\bar{\delta}$ is different from $\bar{\gamma}$; then in particular the tangent vectors to $\bar{\gamma}$ and $\bar{\delta}$ at $\bar{e}$ must determine an angle different from zero. Let $b^{+}$denote a point with positive $s$-value on $\bar{\delta}$, and $b^{-}$a point with negative $s$-value on $\bar{\gamma}$. It is well known that the triangle inequality holds for $b^{+}, b^{-}$, and $\bar{e}$, that is, $d\left(b^{+}, b^{-}\right)<d\left(b^{+}, \bar{e}\right)+d\left(b^{-}, \bar{e}\right)$, provided $b^{+}$and $b^{-}$are sufficiently close to $\bar{e}$ (see [6]). We choose $b^{+}$and $b^{-}$accordingly; let $d\left(b^{+}, \bar{e}\right)+d\left(b^{-}, \bar{e}\right)-d\left(b^{+}, b^{-}\right)=\eta$; we have then $\eta>0$.

We determine $n$ such that $d\left(\bar{\gamma}\left(s_{n}\right), \bar{e}_{n}\right)<\eta / 3$; the inequality $d\left(\bar{\delta}\left(s_{n}\right), \bar{e}_{n}\right)<\eta / 3$ follows then from the fact that the isometrical transformation by the element $a$ transforms $\bar{\gamma}\left(s_{n}\right)$ into $\bar{\delta}\left(s_{n}\right)$, but has $\bar{e}_{n}$ as fixed point, since $\bar{e}_{n}$ belongs to the center of $\bar{G}$. We consider now the following broken path $\bar{\zeta}$ : from $b^{-}$to $b^{+}$on the shortest geodesic joining those two points, from $b^{+}$to $\bar{\delta}\left(s_{n}\right)$ on $\bar{\delta}$, from $\bar{\delta}\left(s_{n}\right)$ to $\bar{e}_{n}$ on the shortest geodesic, and from $\bar{e}_{n}$ to $\bar{\gamma}\left(s_{n}\right)$ on the shortest geodesic. It is clear that the length of $\bar{\xi}$ is less than the distance between $b^{-}$ and $\bar{\gamma}\left(s_{n}\right)$ as measured on $\bar{\gamma}$-the difference being at least $\eta / 3$. But by construction $\bar{\gamma}$ realizes the shortest distance between any two of its points.

Therefore $\bar{\delta}$ cannot be different from $\bar{\gamma}$. But $a$ being an arbitrary element of $\bar{G}$ this means that $\bar{\gamma}$ is in the center of $\bar{G}$; it follows that 
the torus $T$, the closure of $\gamma$, is in the center of $G$, and $G$ is shown not to be semi-simple, which finishes the proof.

\section{REFERENCES}

1. H. Weyl, Theorie der Darstellung kontinuierlicher halbeinfacher Gruppen durch lineare Transformationen III, Math. Zeit. vol. 24 (1925) p. 380.

2. L. Pontrjagin, Topological groups, Princeton, 1939, p. 271.

3. $\mathrm{H}$. Hopf und $\mathrm{W}$. Rinow, Über den Begriff der vollständigen differentialgeometrischen Fläche, Comment. Math. Helv. vol. 3 (1931).

4. W. Rinow, Über Zusammenhänge zwischen der Differentialgeometrie im Grossen und im Kleinen, Math. Zeit. vol. 35 (1932).

5. St. Cohn-Vossen, Existenz kürzester Wege, Compositio Math. vol. 3 (1936) pp. 441-452.

6. C. Caratheodory, Variationsrechnung und partielle Differentialgleichungen erster Ordnung, Leipzig, Berlin, 1935, pp. 319, 320, 322.

SyRACUSE UNIVERSITY 